\title{
Minimizing the Gap between Expectation and Outcome in Breast Augmentation
}

\author{
Jin Hwang ${ }^{1}$, Chul Hwan Seul ${ }^{2}$ \\ ${ }^{1}$ Department of Plastic and \\ Reconstructive Surgery, Yonsei University \\ College of Medicine, Seoul; ${ }^{2} \mathrm{JW}$ Plastic \\ Surgery Center, Seoul, Korea
}

No potential conflict of interest relevant to this article was reported.

\begin{abstract}
Background Most patients who desire breast augmentation have higher expectations than the outcomes that can be achieved. The purpose of this article is to propose strategies for coping with each patient's expectation, and selecting the most appropriate surgical method.

Methods Data were retrospectively reviewed for 138 women who underwent breast augmentation between July 1, 2012 and June 30, 2014. The augmentation methods were selected based on the patients' expectations. According to each expectation, we recommended the optimal procedure and material for each patient, and performed the augmentation in accordance with this as much as possible. The patients were asked postoperatively whether they were satisfied with their outcomes.

Results Most patients (85\%) were satisfied with their results during the mean postoperative follow-up period of $21.4 \pm 7.6$ months (range, $8-32$ months). The remaining, dissatisfied patients (15\%) accepted their final results after receiving explanations or additional procedures.

Conclusions We classified common expectations of breast augmentation patients into nine categories. To minimize the gap between expectations and outcomes, we preoperatively provided realistic explanations about the limitations of the chosen materials and surgical methods with each patient. We then performed breast augmentation in consideration of the patient's expectations. Consequently, we were able to appropriately deal with each patient's expectations.
\end{abstract}

Keywords Breast implantation, Mammoplasty, Patient satisfaction

\section{INTRODUCTION}

According to data from the Korea Medical Dispute Mediation and Arbitration Agency [1], there were 731 consultations for medical disputes in 2013, which was a 64.6\% increase from 444 in 2012. Of these, cases involving plastic surgery were ranked fourth in frequency. There were several cases in which a surgeon lost a suit despite having obtained informed consent preoperatively. As patients

Received: May 6, 2015 Revised: Jun 14, 2015 Accepted: Jun 16, 2015 Correspondence: Chul Hwan Seul JW Plastic Surgery Center, Samsin Building, 836 Nonhyeon-ro, Gangman-gu, Seoul 06025, Korea.

E-mail: pschseul@hanmail.net

Copyright @ 2015 The Korean Society for Aesthetic Plastic Surgery.

This is an Open Access article distributed under the terms of the Creative Commons Attribution Non-Commercial License (http://creativecommons.org/licenses/by-nc/3.0/) which permits unrestricted non-commercial use, distribution, and reproduction in any medium, provided the original work is properly cited. www.e-aaps.org are becoming more fastidious, achieving their desired results is becoming increasingly difficult.

Most patients who desire breast augmentation have higher expectations for outcomes than can be achieved. They think surgery can provide gorgeous, model-like breasts, and they expect a natural shape, soft texture, impalpable implants, ideal size, deep cleavage, natural movement, symmetric and non-ptotic breasts, invisible scars, fast recovery, and minimal pain. To align expectations and outcomes, plastic surgeons should provide realistic, accurate explanations of outcomes when obtaining informed consent and concentrate on eliminating unrealistic expectations.

Focusing on realistic expectations after explaining the limitations of the procedure, pros and cons of the implants or other procedures, and possible complications, we performed breast augmentation surgery and achieved good results. With this clinical experience, we propose strategies of how to cope with expectations of individual patients and select the most appropriate method of surgery. 


\section{METHODS}

Data from 138 women who underwent breast augmentation between July 1, 2012 and June 30, 2014 were reviewed retrospectively. Their mean ( \pm standard deviation $[\mathrm{SD}])$ age was $36.2 \pm 8.1$ years (range, 23-51 years). The mean follow-up period was 21.4 \pm 7.6

Table 1. Classification of patient expectations for breast augmentation surgery

\begin{tabular}{ll}
\hline Number & \multicolumn{1}{c}{ Expectation } \\
\hline 1 & Natural shape \\
2 & Soft texture and impalpable implant \\
3 & Satisfactory volume \\
4 & Deep cleavage \\
5 & Natural movement \\
6 & Symmetric breasts \\
7 & Non-ptotic breasts \\
8 & Invisible scar \\
9 & Fast recovery and minimal pain \\
\hline
\end{tabular}

months (range, 8-32 months). Cases using fat injection were included only if $>2$ injections were performed. We queried each pa-

Table 2. Summary of surgical procedures and materials ( $n=138$ )

\begin{tabular}{lc}
\hline Procedure or material & Number $(\%)$ \\
\hline Incision & \\
Axilla & $90(65)$ \\
Inframammary fold & $42(30)$ \\
Periareolar & $6(4)$ \\
Plane & \\
Dual-plane Type I & $117(85)$ \\
Dual-plane Type II & $15(11)$ \\
Subfascial & $6(4)$ \\
Material & \\
Implant only & $62(45)$ \\
Fat only & $42(30)$ \\
Combination & $34(25)$ \\
Implant & \\
Anatomic & $97(70)$ \\
Round-textured & $35(25)$ \\
Round-smooth & $6(4)$ \\
\end{tabular}

Values are presented as number $(\%)$.
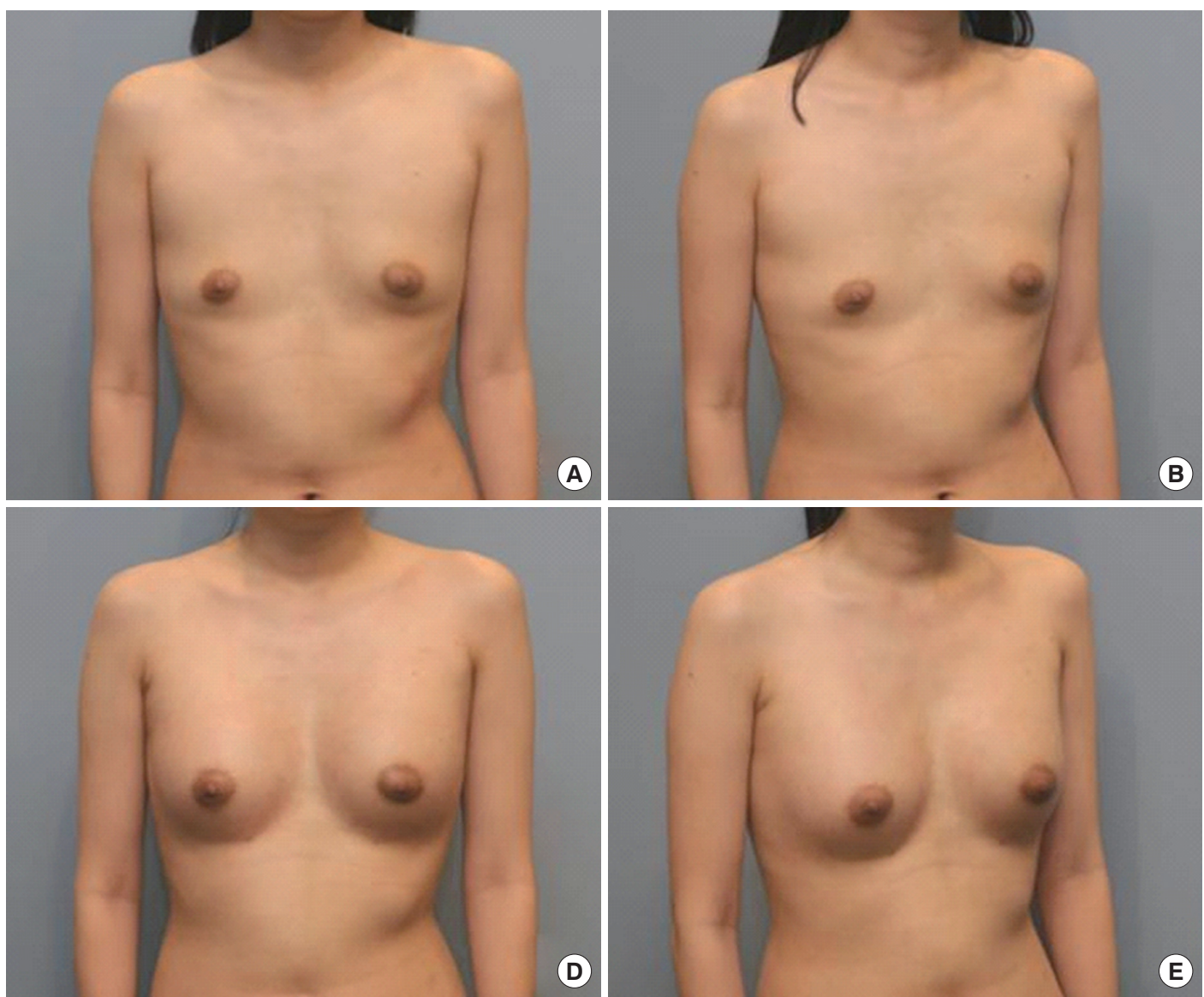
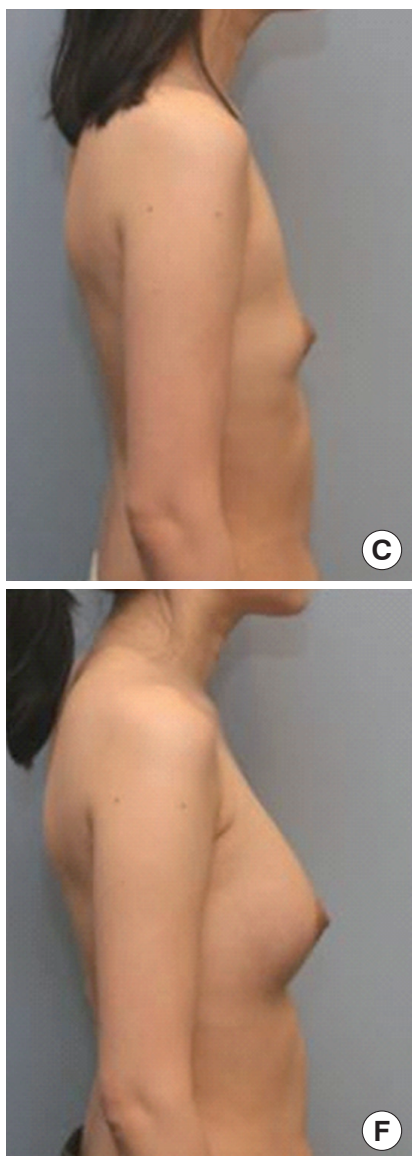

Fig. 1. A 27-year-old nullipara patient underwent Type I dual plane breast augmentation (Silimed 320 MD / 270 LO) and additional fat grafting. $(A, B, C)$ Preoperative view. (D, E, F) Postoperative view 6 months after surgery. 
tient regarding her main expectations. According to our clinical experience, we classified expectations into nine categories (Table 1). For each expectation, we recommended an optimal procedure and material, and followed these recommendations as much as possible. We recommended simultaneous fat injections for all patients undergoing implant-based augmentation and performed injections in those who agreed.

To minimize the gap between expectations and outcomes, we gave each patient reliable estimates about how closely the surgical method could achieve her goals. We fully explained the limitations of each material and procedure, and received preoperative written informed consent indicating agreement with the planned operation. Postoperatively, the patients were asked to rate their satisfaction with the results as very satisfied, satisfied, unsatisfied, or very unsatisfied.

\section{RESULTS}

We used the optimal material and method of breast augmentation based on each patient's expectations and physical characteristics. Most patients (85\%) were satisfied with their results during the mean postoperative follow-up period of 21.4 \pm 7.6 months (range, 8-32 months; Table 2). Below, we describe several cases illustrating our approach to each expectation.

\section{Natural shape}

We explained to patients that breast augmentation would not produce a perfect breast and that the postoperative breast and chest wall shape would reflect the preoperative breast. Making a pocket in the dual plane, using an anatomical rather than a round implant, and performing fat injections helped achieve a natural shape.

For a 27-year-old nullipara patient with constricted breasts and pectus excavatum, we performed augmentation mammoplasty (Type II dual plane) plus fat injections. The shape of the breasts and chest wall was improved, but the original shape was not completely eliminated. This had been explained preoperatively, so the patient accepted the results (Fig. 1).

\section{Soft texture and impalpable implant}

We recommended fat injection for patients wanting perfectly natural, soft-textured breasts and impalpable implants. We informed patients that repeat fat injections may be necessary because suffi-
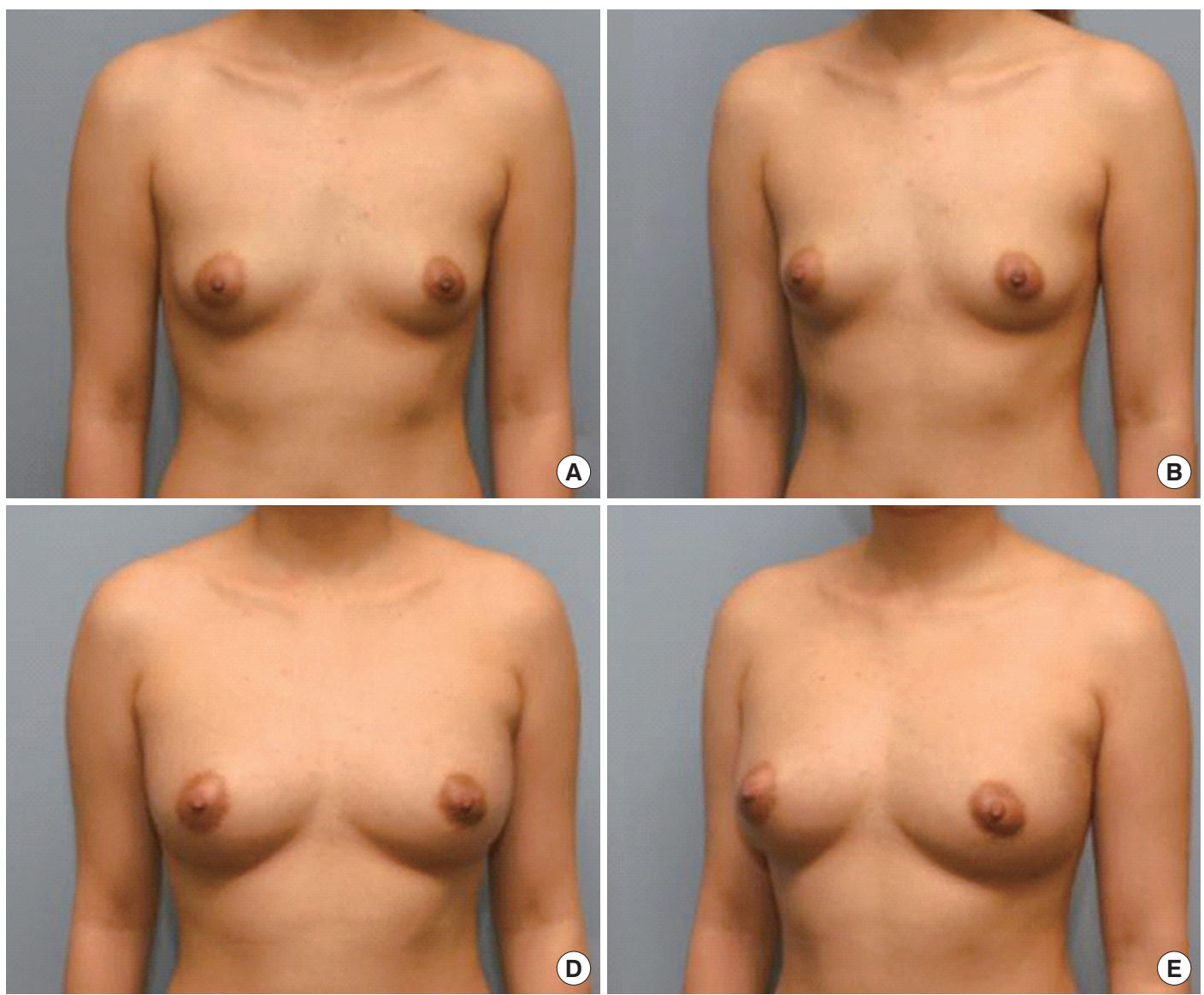

(B)

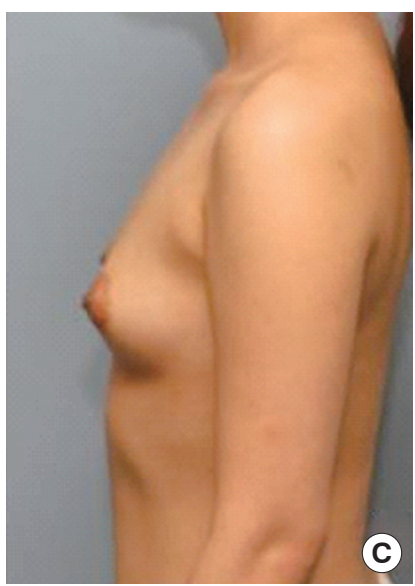

(E)

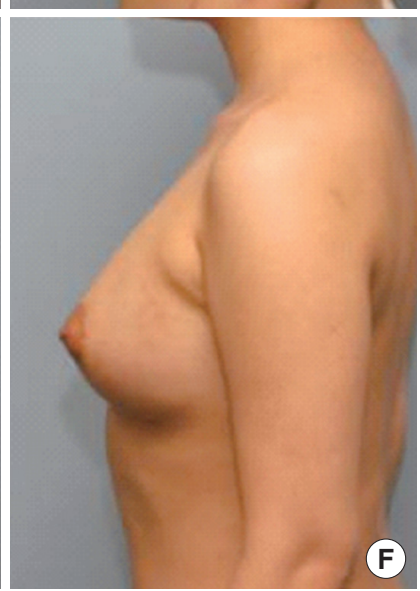

Fig. 2. A 30-year-old nullipara patient who wanted perfect natural texture and an impalpable implant was treated with two sessions of fat injections. (A, B, C) Preoperative view. (D, E, F) Final postoperative view 8 months after the second operation. 
cient volume expansion may not be achievable with initial injections. In case of using implant, we explained that all implants are palpable and that adding fat injections may be necessary to increase the surrounding soft tissue thickness. Because preventing capsular contracture is important in obtaining a soft breast, we tried to achieve meticulous hemostasis, used abundant irrigation and a no-touch surgical technique, and prescribed a leukotriene-receptor antagonist (e.g., montelukast) for 1 to 2 months postoperatively.

For a 30-year-old nullipara woman desiring natural breast texture and impalpable implants, we performed fat injections without implants (Fig. 2). For a 36-year-old multipara patient with very thin soft tissue who wanted a soft texture, we performed combination breast augmentation using implants and fat injections (Fig. 3).

\section{Satisfactory volume}

Many patients report their desired breast size as a bra cup size, but this is difficult to measure. Instead, we asked patients, "Do you want natural and proportional breasts or breasts as large as possible, with physical conditions permitting?" We then asked them to select their preference while viewing postoperative pictures of other patients.
When patients wanted large breasts, implants were primarily considered. The chest width was the primary parameter used for selecting the implant. When patients with a narrow chest wanted large breasts, we used a high-projection implant. However, patients were informed that when implants are too large, palpability and rippling are more severe. When patients with a short nipple to inframammary fold (IMF) distance wanted large breasts, we used the dual plane method with form-stable implants, combined with fat injections to minimize the risk of double-bubble deformity [2]. Because shorter IMF increases the likelihood of a double-bubble deformity, the importance of a proper-sized implant was explained to the patient. Most patients were satisfied with their postoperative breast size.

\section{Deep cleavage}

In patients with a wide sternum or separated breasts, it is difficult to achieve deep cleavage. In these situations, partial detachment of the sternal origin of the pectoralis major was considered. However, excessive detachment must be avoided, as this can produce a palpable implant and synmastia. For patients with separated breasts, we used implants with sufficient diameter to cover half of the chest.
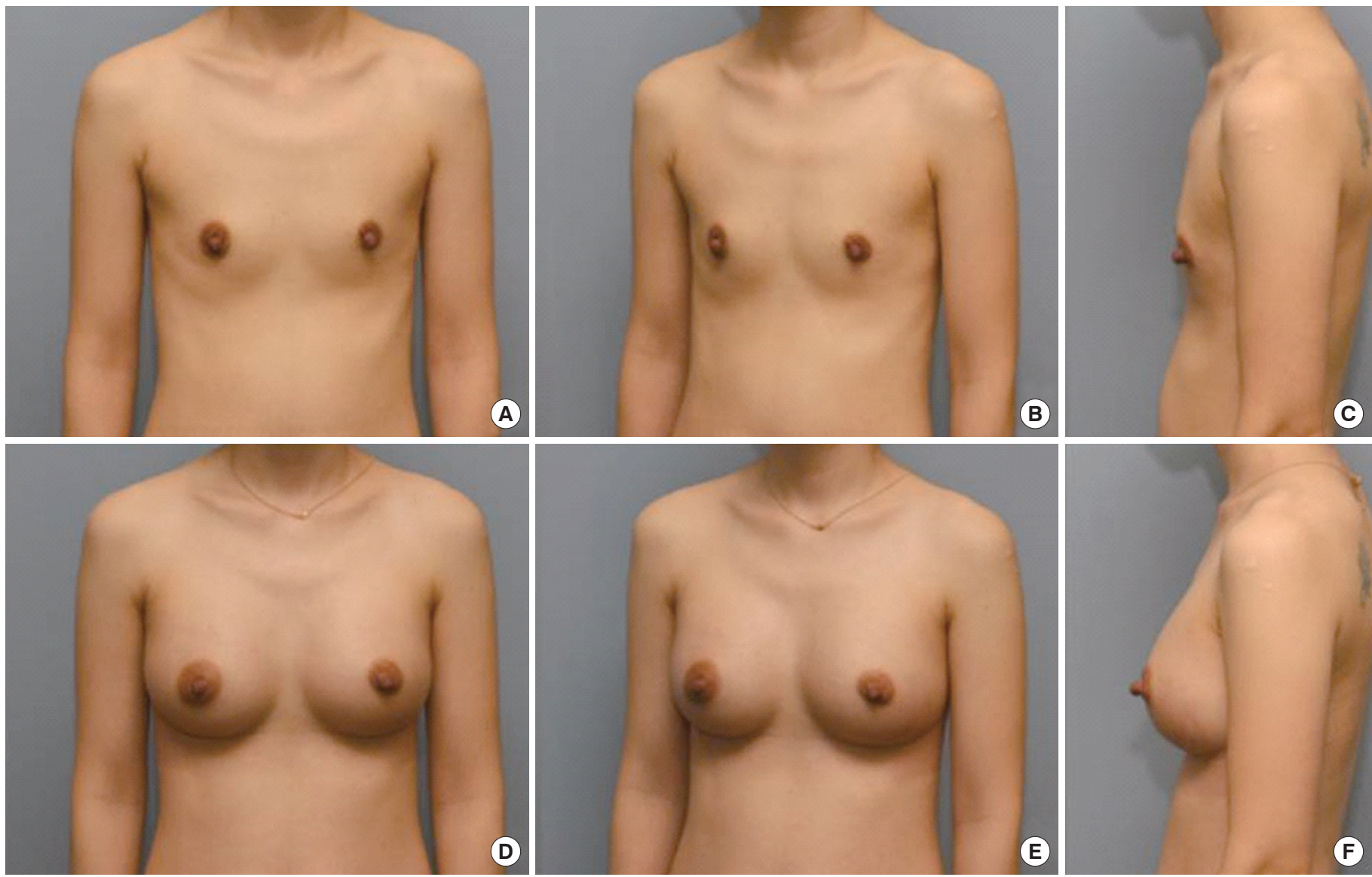

Fig. 3. A 36-year-old multipara patient who wanted better texture underwent Type I dual plane breast augmentation (Polytech TMS $255 \mathrm{~mL}$ ) and simultaneous fat injection to compensate for her thin soft tissue. (A, B, C) Preoperative view. (D, E, F) Postoperative view 10 months after surgery. 

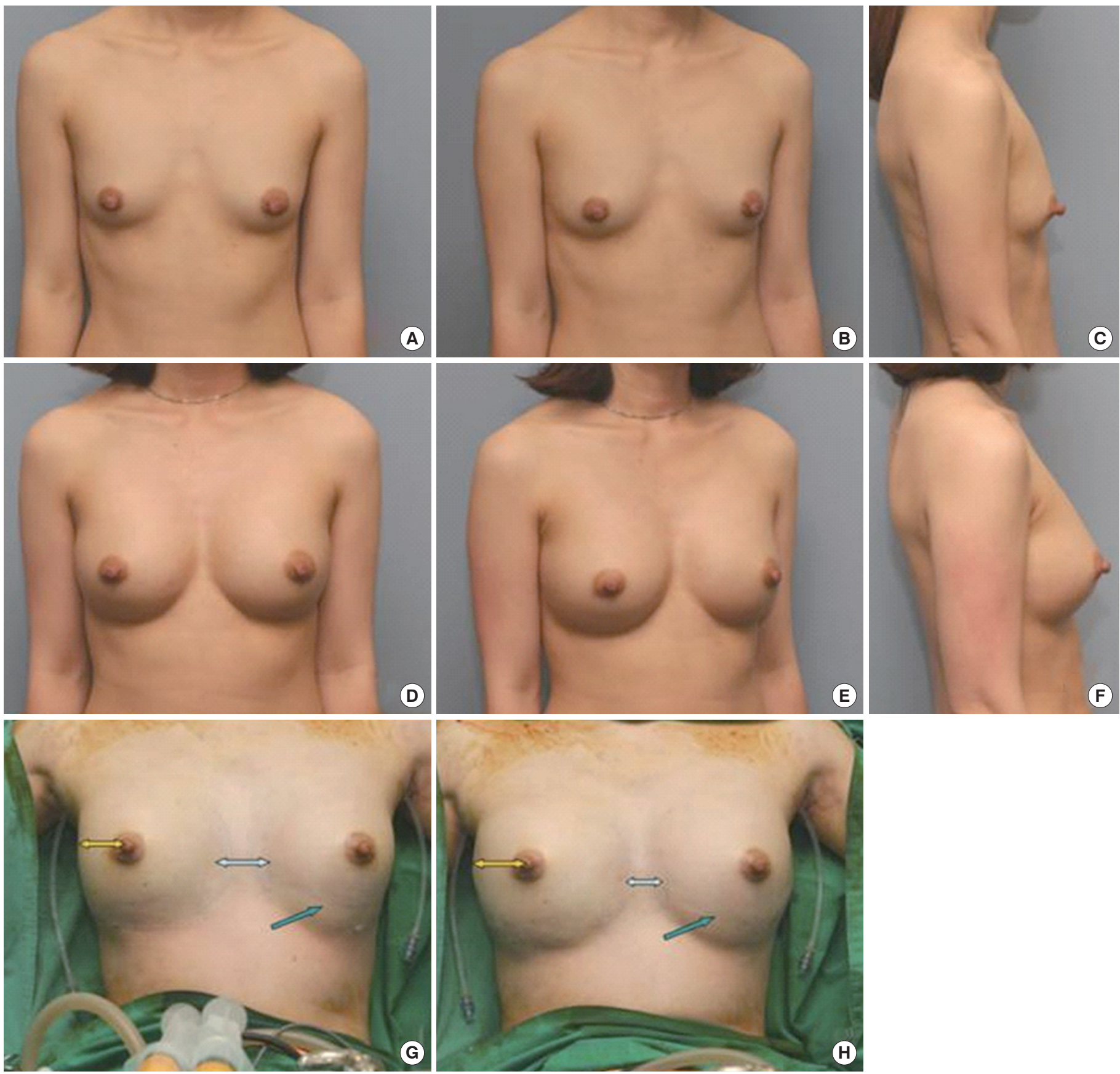

Fig. 4. A 26-year-old nullipara patient treated with Type I dual plane augmentation (Polytech TMS $280 \mathrm{~mL}$ ) and fat injection. (A, B, C) Preoperative view. (D, E, F) Postoperative view 11 months after surgery. (G) Wide gap between the two breasts and visible old IMF before fat injection. (H) Less visible old IMF and improved cleavage after fat injection.

Fat injections were added for better cleavage.

After pocket dissection and insertion of a sizer in a 26-year-old nullipara patient with thin soft tissue, a wide sternum, lower pole constriction, and a large internipple distance, we noticed a wide gap between the breasts, laterally deviated nipples, and a visible old IMF. After fat injection, the cleavage was improved, nipples appeared less separated (because of lateral enhancement), and the old IMF was not visible (Fig. 4). In our patients, grafted fat often contracted during the first 3 to 4 months after injection, but after that, breast volume was relatively well-maintained in most patients for $>1$ year of follow-up.

\section{Natural movement}

Patients were informed that natural breast movement depends on 

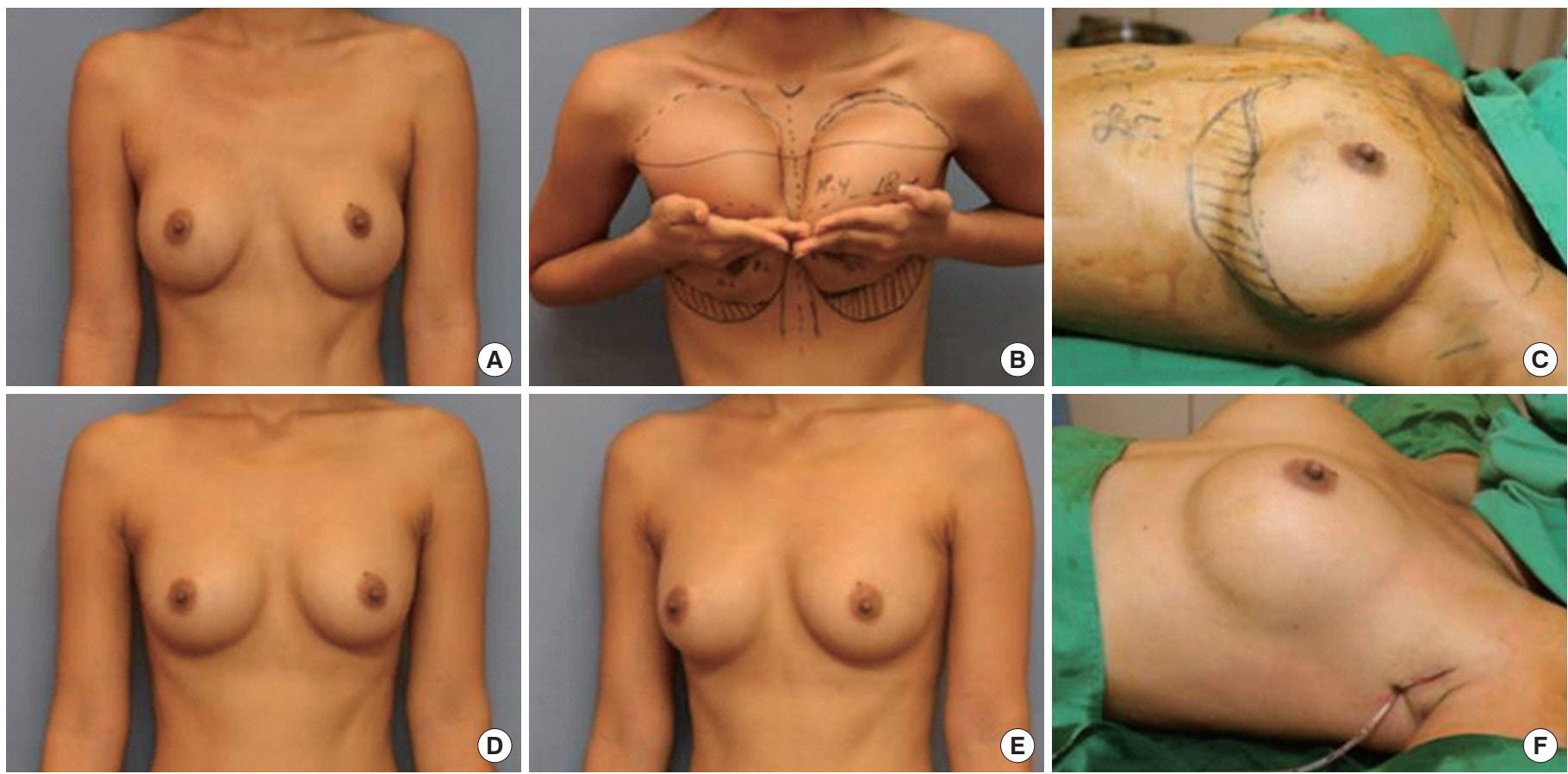

Fig. 5. A 27-year-old nullipara patient with malposition of a previously inserted smooth implant and separate movement was treated with endoscopic transaxillary capsule cauterization and the implant was exchanged for a textured implant (Style 115, 272 mL). (A, B) Preoperative view. (C, F) Intraoperative view. (D, E) Postoperative view 10 months after surgery.

the amount of parenchyma. Fat injection was beneficial to increase parenchymal thickness. For implant-based augmentation of severe parenchymal hypoplasia, we considered the pros and cons of different implants. Smooth implants are sometimes assumed to provide more natural movement and spreading, but they tend to move separately from the patient's parenchyma, resulting in lateral displacement in the supine position. They are also more likely to become malpositioned. Textured implants are less movable, producing slightly more rippling but a more stable result. Round implants are softer and more transformable but cause more rippling, whereas form-stable implants are firmer but produce less rippling and more tissue expansion. We should let the patients understand that there always exist demerits whichever implant is chosen.

We often performed combination breast augmentation to achieve natural movement. A 27-year-old nullipara patient had extremely unnatural movement after previous breast augmentation with smooth implants. She had inferior malposition, a left wide pocket, and lateral displacement when supine. She underwent capsule cauterization and replacement with textured implants (Fig. 5).

\section{Symmetric breasts}

We informed patients that human bodies are not perfectly symmetric, and size differences could not be completely corrected. For patients with different breast sizes, we used different sizes of implants and/or volumes of injected fat in each breast. Different IMF levels were adjusted by lowering the IMF by different amounts dur- ing dissection or using a different chest wall IMF fixation level during IMF incision. With deep IMFs, lowering the IMF during dissection can cause a double-bubble deformity. In these instances, we used different IMF fixation levels: The IMF was fixed slightly lower on the chest wall of the higher IMF side, and fixed higher on the opposite breast. For patients with slightly different ptosis grades, different types of dual plane type were helpful. With severe differences, different degrees of mastopexy were needed.

For a patient with right anterior thoracic hypoplasia, we performed combination breast augmentation with round-textured implants and fat injections. A high-profile $300 \mathrm{~mL}$ implant was inserted on the right and a low-profile $210 \mathrm{~mL}$ implant with similar diameter was used on the left. Different volumes of fat were injected into different areas (Fig. 6). For a patient with left breast parenchymal hypoplasia who did not want implants, we conducted two fat injection sessions (Fig. 7). For a patient with different ptosis grades, different degrees of crescent mastopexy were performed in each breast (Fig. 8).

\section{Non-ptotic breast}

Patients with small, ptotic breasts expected simultaneous correction of the size and ptosis. For mild ptosis, dual plane pockets and anatomical implants were useful. For moderate or severe ptosis, augmentation mastopexy was necessary. Some people assume that round implants are preferable for ptosis, as they may fill in the sunken upper pole, but the most projecting point of a round implant is 

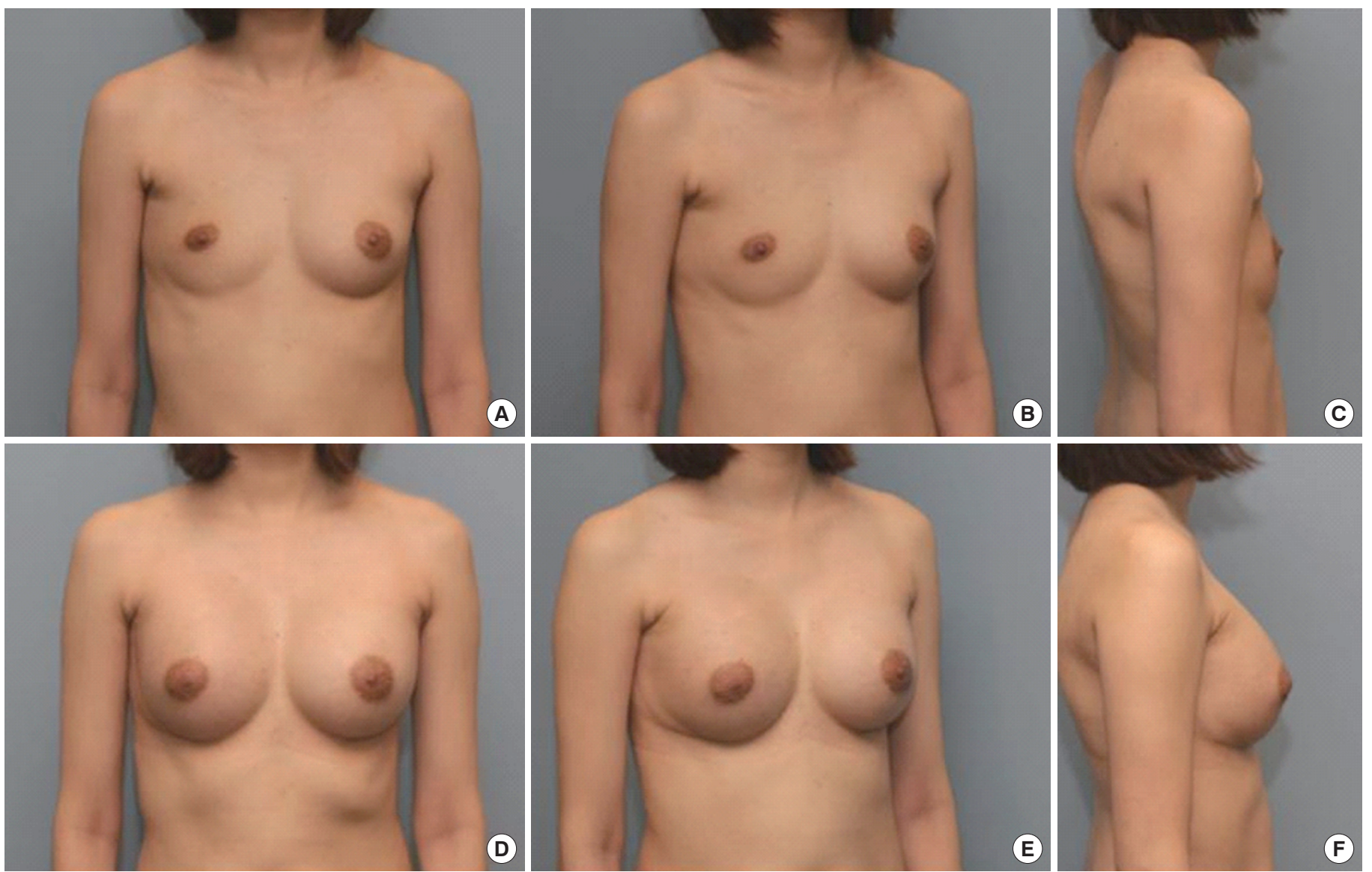

Fig. 6. A 26-year-old nullipara patient with right anterior thoracic hypoplasia was treated with combination augmentation $(S t y l e 120,300 \mathrm{~mL}$ on the right and Style 110, $210 \mathrm{~mL}$ on the left) by an endoscopic transaxillary approach with a Type I dual plane method and fat injections (153 $\mathrm{mL}$ on the right and $66 \mathrm{~mL}$ on the left). (A, B, C) Preoperative view. (D, E, F) Postoperative view 10 months after surgery.

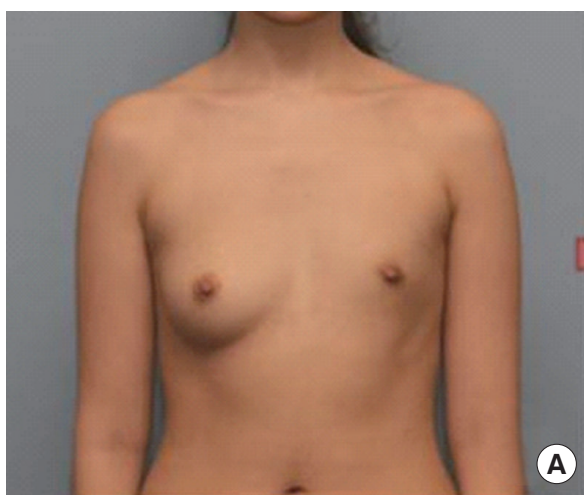

Preop.

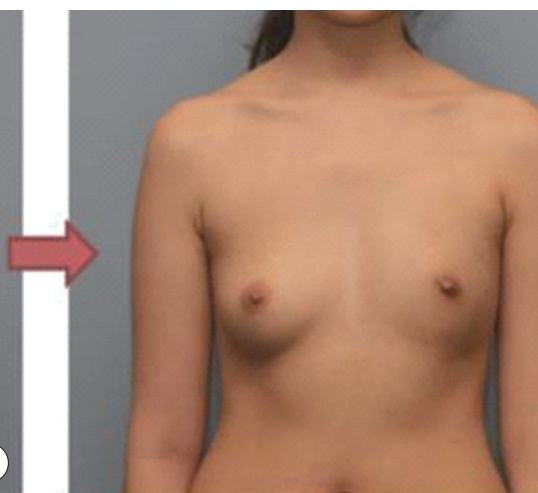

1st fat injection POD $4 \mathrm{~m}$

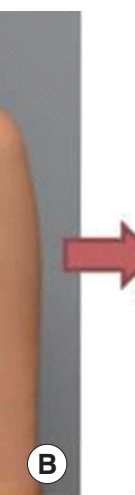

B

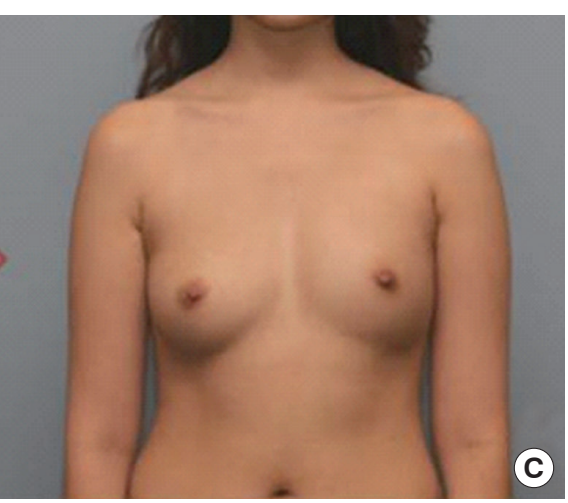

2nd fat injection POD $2 \mathrm{~m}$

Fig. 7. A 25-year-old nullipara patient underwent staged fat grafting. (A) Preoperative view. (B) Four months after the first injections (270 mL in the left breast only). (C) Two months after the second injections (141 mL on the right and $243 \mathrm{~mL}$ on the left). Preop., preoperation; POD, postoperative day.

at its center, which can lead to waterfall deformity if used for severely ptotic breasts. Conversely, the most projecting point of an anatomical implant is on the lower part, so the implant can expand the lower pole and lift up the nipple areolar complex. Thus, for sunken breasts without ptosis, round implants will suffice, but for ptotic breasts, anatomical implants are preferred. 

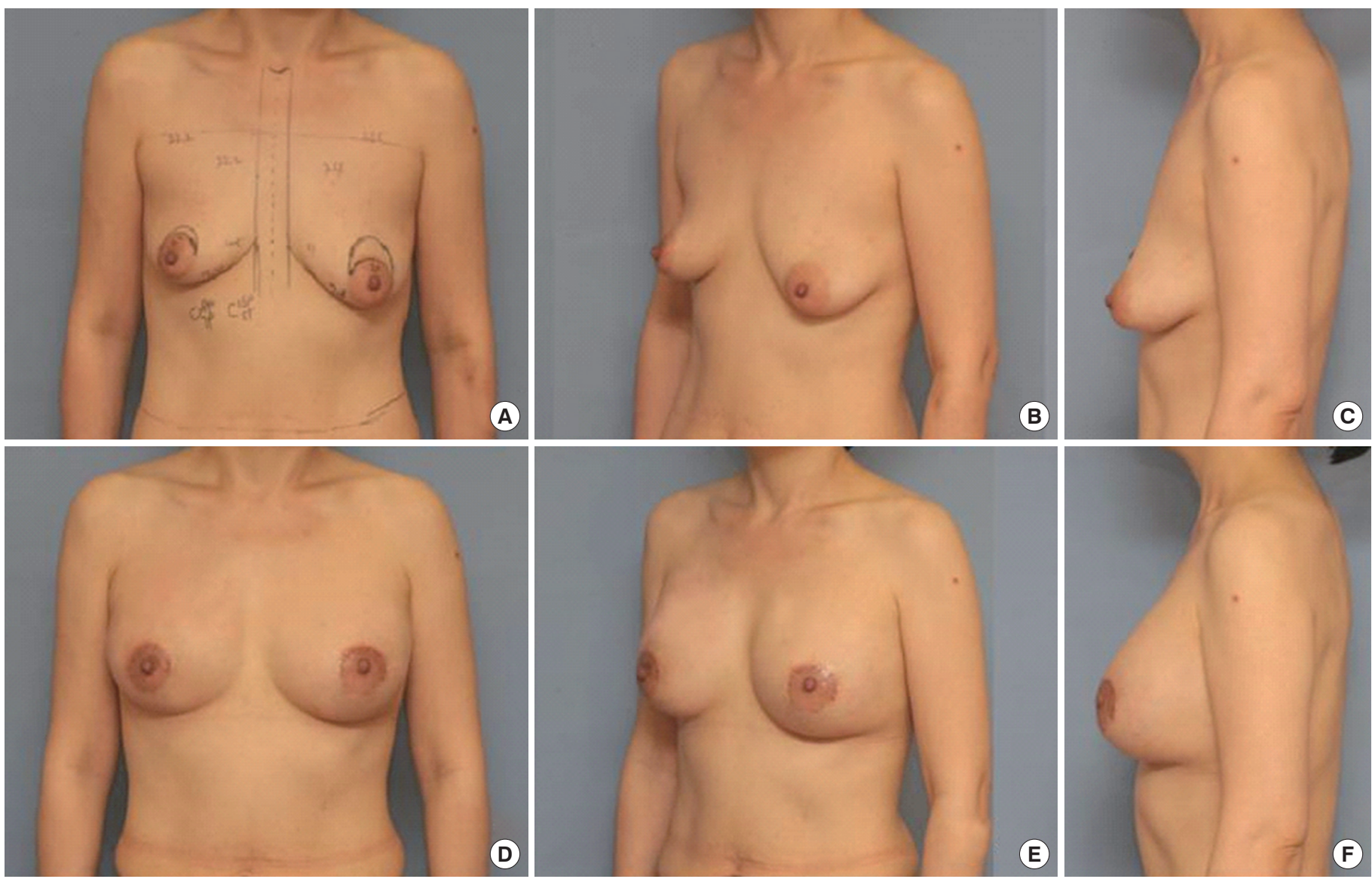

Fig. 8. A 44-year-old multipara patient with different ptosis grades on each side was treated with different degrees of crescent mastopexy and augmentation (Siltex moderate plus $300 \mathrm{~mL} / 275 \mathrm{~mL}$ in the subfascial plane). (A, B, C) Preoperative view. (D, E, F) Postoperative view 2 weeks after surgery.

For a patient with mild ptosis, Type II dual plane augmentation using anatomical implants was performed (Fig. 9). For severe ptosis, we obtained a satisfying result with Type I dual plane augmentation and simultaneous periareolar mastopexy (Fig. 10).

\section{Invisible scar}

Skin tension in the axilla is low, so scarring is less apparent in that area. When using an axillary incision, we made the incision in the crease of the axillary apex. Most Asian patients assume that an IMF incision will produce severe scarring; however, when an IMF incision is used for patients with a deep IMF, scarring is minimal. If a zig-zag periareolar incision is used for patients with a sufficient areolar diameter, it leaves a minimal scar but is prone to parenchymal injury, bacterial contamination, a depressed scar, and areolar distortion. Thus, we used a periareolar incision only when it was absolutely necessary. Supportive taping, silicone gel sheets, scar-reducing ointment, hydroquinone, and LASER therapy can help produce inconspicuous scars.

We generally used an axillary approach for nullipara patients with tight breasts and shallow IMFs. For patients with a deep IMF or less skin tension around the IMF, IMF incisions were used and the resulting scars were almost inconspicuous (Fig. 11). Periareolar incision was used only when patients had previous surgery through a periareolar incision or required a mastopexy.

\section{Fast recovery and minimal pain}

With an axillary approach, endoscopic dissection produces faster recovery and less pain than conventional blind blunt dissection [3]. When an axillary incision was used, the entire dissection was performed via endoscopy and electrocautery. IMF or periareolar approaches also led to faster recovery than the conventional axillary approach. For postoperative pain control, we instilled local anesthetic into the pocket and prescribed oral analgesics. Postoperative pain was tolerable in all patients.

\section{Expectations for revision surgery}

Revision surgery patients have similar expectations, although their expectations tend to be more realistic because of their previous experiences. To improve softness, existing implants can be replaced by softer implants; capsular contracture, if present, should be cor- 

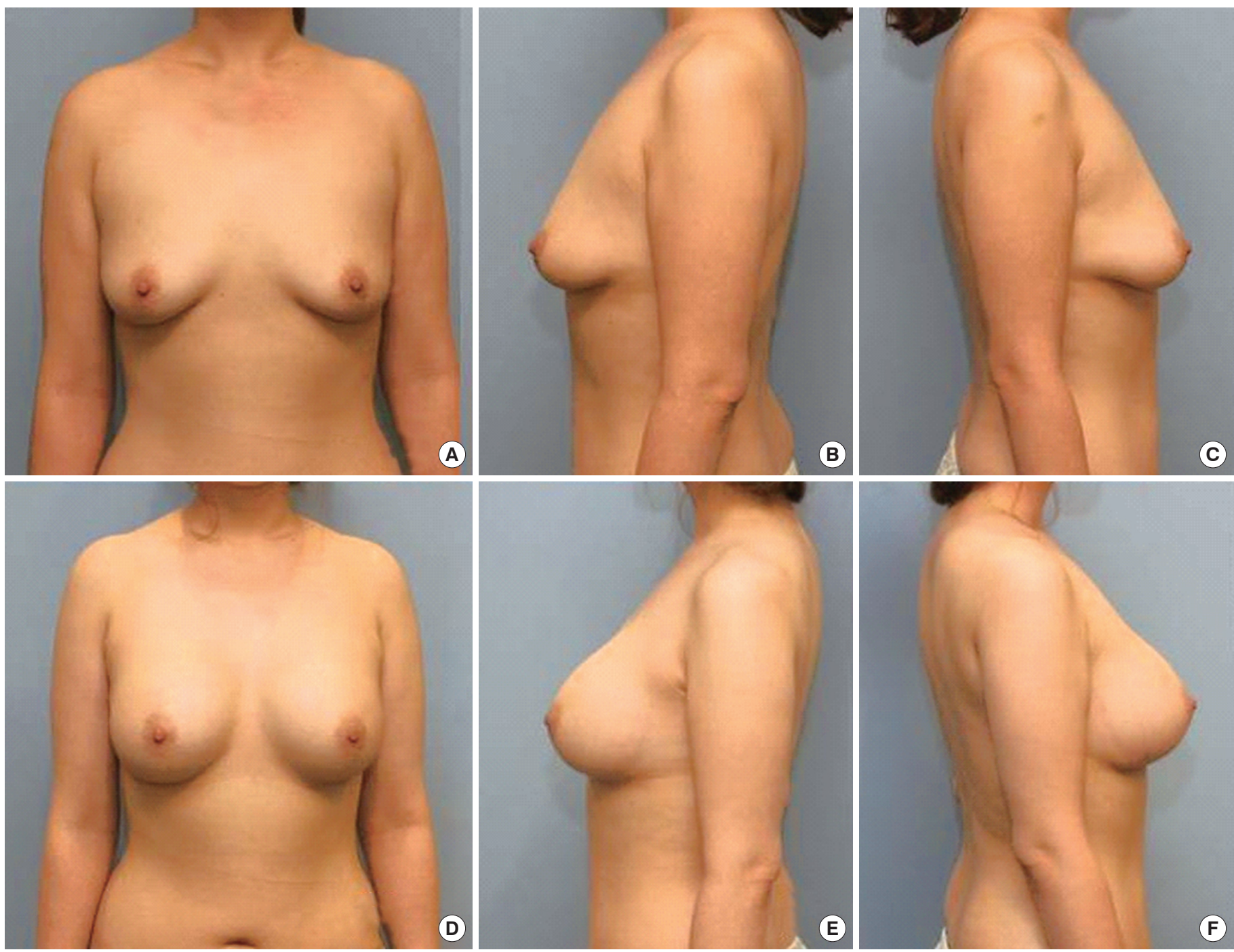

Fig. 9. A 34-year-old nullipara patient with mild ptosis was treated with Type II dual plane augmentation using an intramammary fold approach (right: Sebbin TL 330 mL, left: Sebbin TL 330 mL). (A, B, C) Preoperative view. (D, E, F) Postoperative view 10 months after surgery.

rected. If patients no longer want an implant, it can be explanted and fat injected as a replacement. Simultaneous mastopexy can also be performed if necessary. If the soft tissue is too thin and the implant is in the subglandular plane, pocket conversion to the subpectoral plane is an option. When the capsule in the subglandular plane is thin, we do not remove the implant. If a thick capsule interferes with soft tissue expansion, it may be necessary to perform capsulotomy, or partial or total capsulectomy [4]. If an implant is in the subpectoral plane, an acellular dermal matrix graft or fat injection should be considered [5]. If an implant is malpositioned, it should be repositioned.

A 37-year-old multipara woman with previous breast augmentation surgery had a grade IV capsular contracture and superior malposition of an implant in the subpectoral plane. To meet her expectations of a natural shape and soft texture, we performed total capsulectomy via a transaxillary endoscopic approach and reposi- tioned her implant (Fig. 12).

\section{Management of the unsatisfied group}

There were 21 patients in the dissatisfied group (Table 3). Size dissatisfaction was the most common complaint, noted by nine patients. Three of these patients had implants and six had fat injection alone. For one implant patient, the implant was exchanged for a bigger one. The two other implant patients accepted their results after we explained that the chosen method was the most proper way to minimize complications for their specific breast conditions. In the fat injection cases, we explained the limitations of fat grafts and most women accepted their results. For the two who did not, we performed augmentation with an implant and a third fat injection. Five patients were dissatisfied with the texture. Of these, three who complained of rippling underwent additional fat injections, and one with a left breast grade III capsular contracture underwent 

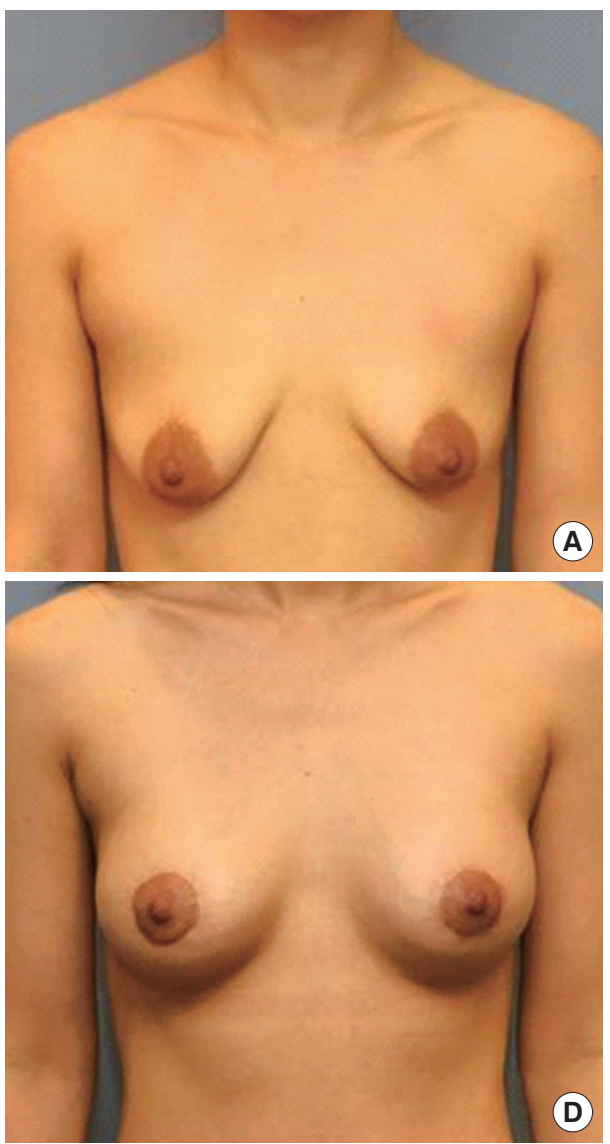
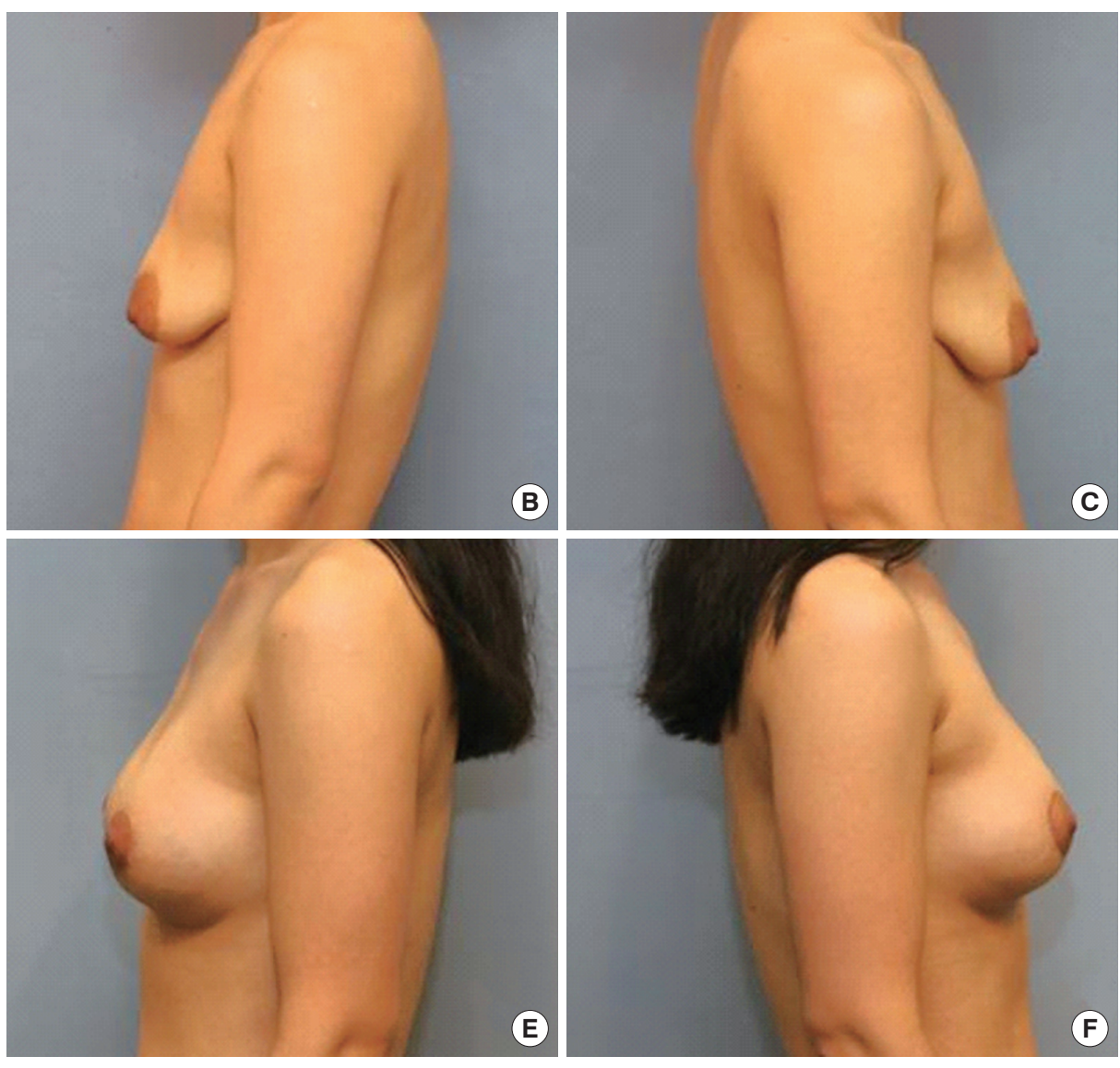

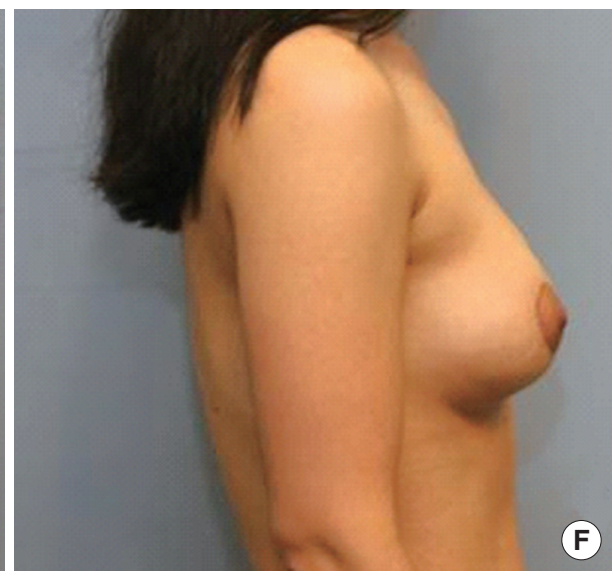

Fig. 10. A 43-year-old multipara patient with severe ptosis was treated with augmentation and periareolar mastopexy (Type I dual plane, right: Style 115-203, left: Style 115-222). (A, B, C) Preoperative view. (D, E, F) Postoperative view 23 months after surgery.

revision surgery involving total capsulectomy and new implant insertion. The remaining patient did not want further surgery. The one patient who was dissatisfied with breast shape initially had bilateral ptosis. She complained of minimally undercorrected ptosis, but it was not severe enough to warrant additional surgery. For the two women dissatisfied with breast asymmetry, malpositions were noted. Camouflage fat injection was performed for the patient with mild superior malposition. Capsule cauterization and capsulorrhaphy were performed for inferior malposition in the other patient. Two patients dissatisfied with breast movement accepted the results after we explained that unnatural movement arose from the inherent properties of anatomic form-stable implants and their thin parenchyma. Patients who complained of hypertrophic scars accepted the results after receiving two to three intra-scar triamcinolone injections. Thus, all dissatisfied patients accepted their final results after explanations or additional procedures.

\section{DISCUSSION}

Over 11 billion cosmetic surgical procedures were performed worldwide in 2013, almost double the number performed in 2010 [6].
Korea was ranked seventh in the total number of aesthetic procedures and first in the number of operations per 10,000 persons in 2011 [6]. Worldwide, the most popular cosmetic surgical procedure was breast augmentation in 2013 [6].

As the interest in breast augmentation increases, patients' expectations also increase. Most patients have high expectations that cannot be fully achieved. There is a gap between expectations and surgical outcomes, and efforts should be made to minimize this gap.

In this study, we described our approach of preoperatively explaining all the limitations of materials and surgical methods to reduce unrealistic expectations, and then performing breast augmentation using the most appropriate procedures for each expectation. Accordingly, most patients (85\%) were satisfied with their outcomes. The remaining, dissatisfied patients accepted the final results after explanations or additional procedures. The six patients who underwent augmentation with only fat injections comprised a large portion of the size dissatisfaction group. Although many techniques have been introduced to enhance cell survival in fat grafts, their use in breast augmentation continues to generate size complaints because of unpredictable resultant volume estimation. Thus, patients should be warned preoperatively about relatively poor expansion 

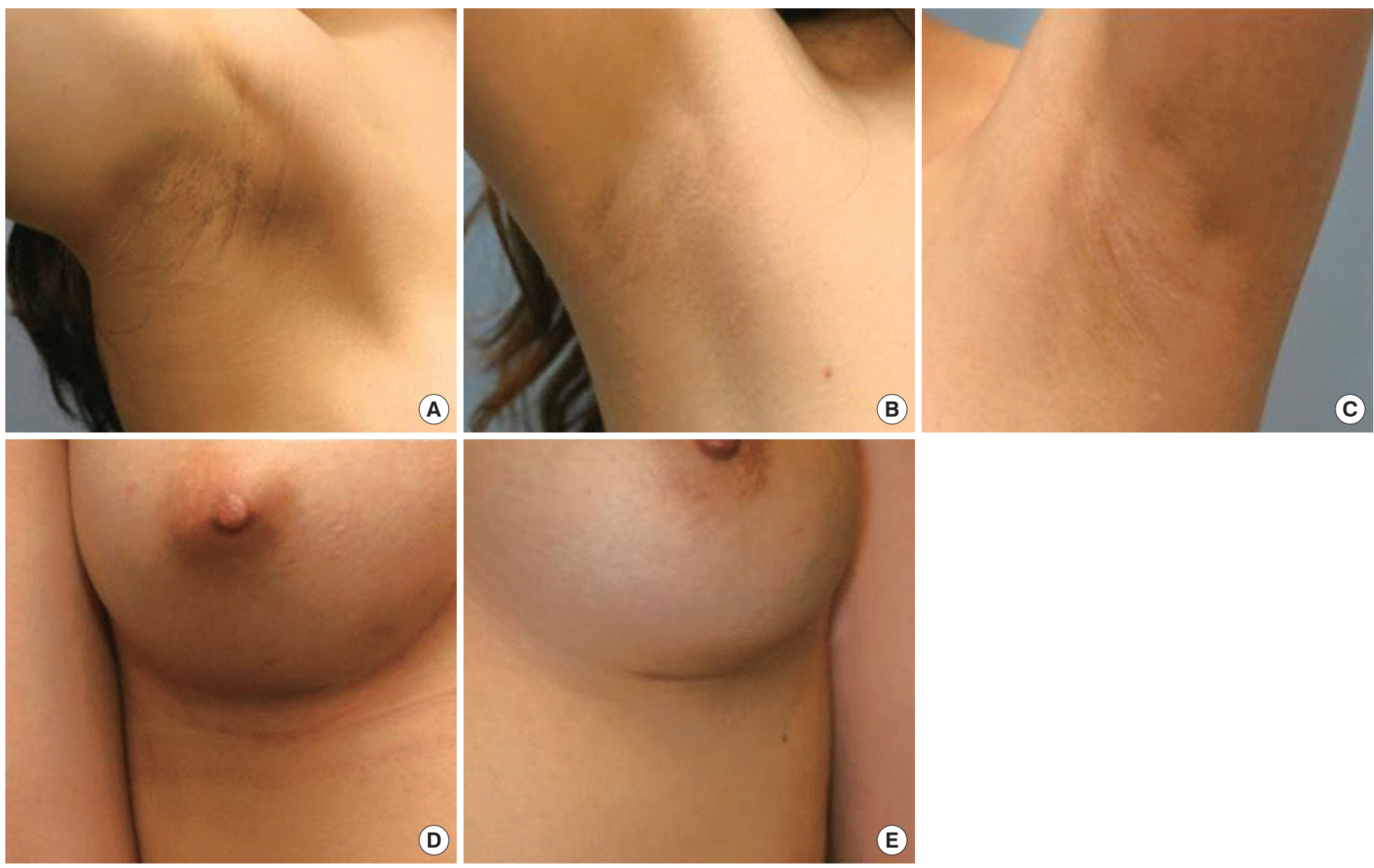

Fig. 11. Postoperative scar. (A, B, C) Axillary scar. (D, E) Intramammary fold scar.

outcomes of breast augmentation with fat injections.

We generally used dual plane breast augmentations. The usefulness of this augmentation for optimizing implant-soft tissue relationships has been previously discussed. Its advantages include an optimal lower pole breast volume, less noticeable implant, and lower risk of capsular contracture [7]. The dual plane method not only expands the lower pole, but it lifts the soft tissue along the pectoralis major muscle contracture, making it particularly useful for ptosis correction. Furthermore, by using different types of dual plane methods, we successfully managed slight differences in ptosis grades between both breasts.

Composite breast augmentation is an implant-based augmentation with supplementary fat injections in the subcutaneous layer or pre-pectoral plane, which produces a more natural look [8]. We used this method for patients with thin soft tissue, asymmetry, chest wall deformity, and "separated-breasts" deformity. Breast augmentation using autologous fat injections is extremely useful for achieving symmetry and a natural shape and texture, and using staged fat injections, we attained considerable volume augmentation [9]. Thus, when used alone or combined with implants, fat injection is a highly useful method to enhance patient satisfaction.

Implant selection is also important, and it is necessary to explain to patients that no implant will be as natural as their existing breast. Textured implants are less movable and cause slightly more rippling than smooth implants, but they can provide more stable results; therefore, we used textured implants in most revision operations. For patients with mild ptosis, anatomical form-stable implants are more effective than round implants because the most projecting part of anatomical implants is on the lower part. This produces greater expansion of the lower pole, thus pushing up the nipple areolar complex. For moderate or severe ptosis, mastopexy should be performed with augmentation.

To obtain invisible scars, the transaxillary approach showed good results. It is very popular in Asian countries because of minimal scarring. Using endoscopy has the advantages of precise dissection, improved hemostasis, and less tissue injury, resulting in less pain and faster recovery, compared to the conventional blind blunt axillary approach [3]. For patients with deep IMF, an IMF approach can be a good choice.

In recent years, there has been an increase in foreigners visiting Korea for medical tourism, and as Korean plastic surgery becomes more renowned, more foreign patients visit plastic surgery clinics in the country. However, because of the language barrier and irregular follow-up, communication and postoperative management 

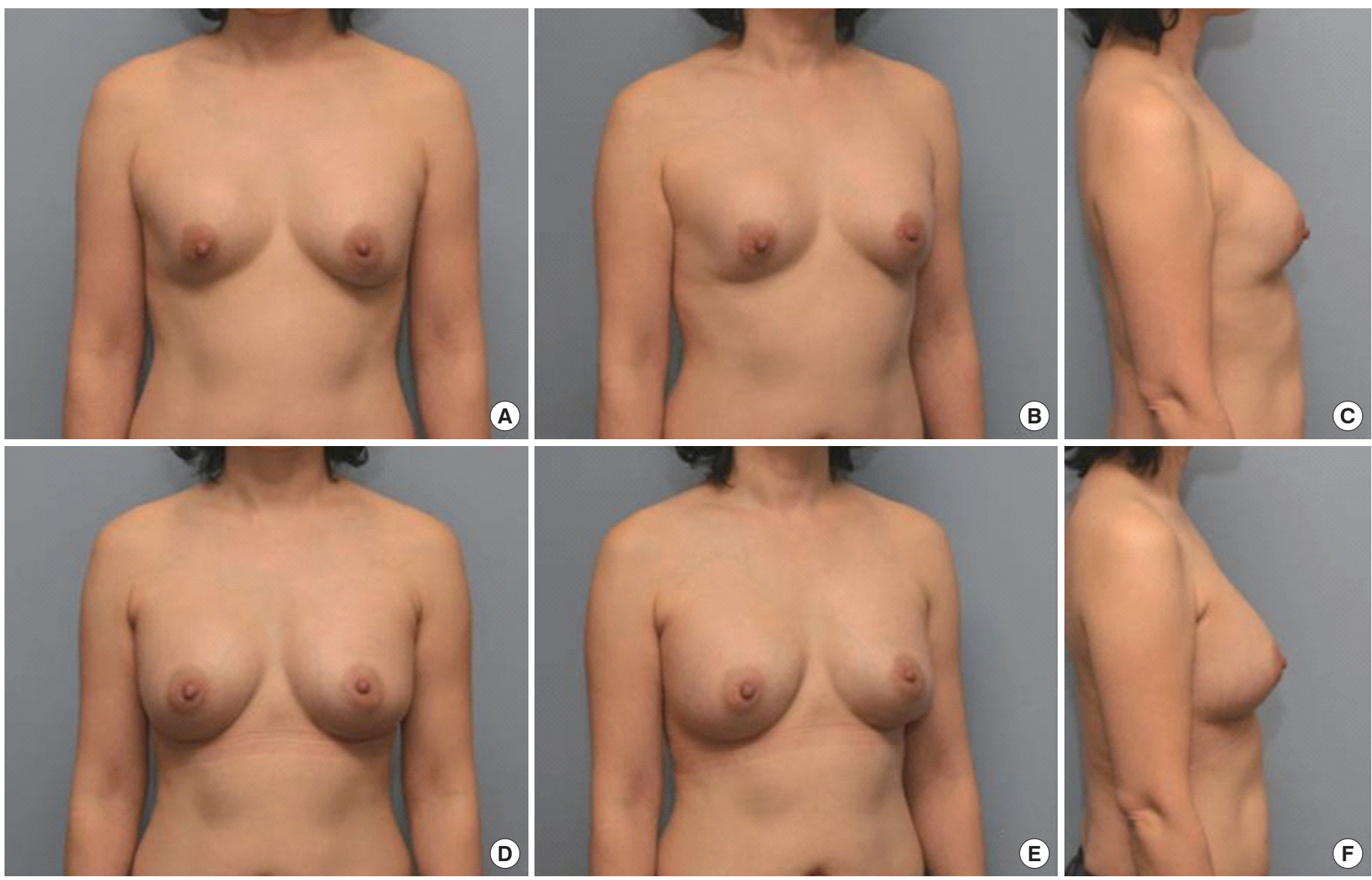

Fig. 12. A 37-year-old multipara patient was treated by transaxillary endoscopic total capsulectomy. The shape and softness were improved as desired. (A, B, C) Preoperative view. (D, E, F) Postoperative view 9 months after surgery.

Table 3. Outcomes and causes of dissatisfaction

\begin{tabular}{lc}
\hline Outcome or cause & Number $[\%]$ \\
\hline Outcome & \\
Very satisfied & $84(61)$ \\
Satisfied & $33(24)$ \\
Dissatisfied & $21(15)$ \\
Very dissatisfied & $0(0)$ \\
Cause of dissatisfaction & \\
Size & $9(6.5)$ \\
Texture & $5(3.6)$ \\
Movement & $2(1.4)$ \\
Asymmetry & $2(1.4)$ \\
Scar & $2(1.4)$ \\
Shape & $1(0.7)$ \\
\hline
\end{tabular}

Values are presented as number $(\%)$.

can be impaired. To cope with these patients, we should choose a simple breast augmentation procedure with a fast recovery, requiring few follow-up visits, and producing predictably good results.

In this study, we classified common expectations of breast augmentation patients into nine categories. To minimize the gap between expectations and outcomes, realistic explanations were dis- cussed preoperatively with the patients about the limitations of the materials and surgical methods. We then performed breast augmentation considering these expectations. This allowed us to appropriately deal with our patients' expectations.

\section{PATIENT CONSENT}

Patients provided written consent for the use of their images.

\section{REFERENCES}

1. Korea Medical Dispute Mediation and Arbitration Agency. Medical dispute mediation and arbitration statistical yearbook 2012. Seoul: Korea Medical Dispute Mediation and Arbitration Agency; 2013.

2. Handel N. The double-bubble deformity: cause, prevention, and treatment. Plast Reconstr Surg 2013;132:1434-43.

3. Seul $\mathrm{CH}$. Endoscopic transaxillary breast augmentation with cohesive gel implant. J Korean Soc Aesthetic Plast Surg 2009;15:24-30.

4. Spear SL, Carter ME, Ganz JC. The correction of capsular contracture by conversion to "dual-plane" positioning: technique and outcomes. Plast Reconstr Surg 2006;118:103S-13S. 
5. Maxwell GP, Gabriel A. Acellular dermal matrix in aesthetic revisionary breast surgery. Aesthet Surg J 2011;31:65s-76s.

6. International Society of Aesthetic Plastic Surgery. ISAPS global statistics. Hanover, NH: International Society of Aesthetic Plastic Surgery, 2013. [cited by 2014 Nov 1]. Available from: http://www.isaps.org/news/ isaps-global-statistics.

7. Tebbetts JB. Dual plane breast augmentation: optimizing implant-softtissue relationships in a wide range of breast types. Plast Reconstr Surg
2001;107:1255-72.

8. Auclair E, Blondeel P, Del Vecchio DA. Composite breast augmentation: soft-tissue planning using implants and fat. Plast Reconstr Surg 2013;132:558-68.

9. Khouri RK, Khouri RK Jr, Rigotti G, et al. Aesthetic applications of Brava-assisted megavolume fat grafting to the breasts: a 9-year, 476-patient, multicenter experience. Plast Reconstr Surg 2014;133:796-807. 